\title{
Redução de aminoácidos em polpas de bacuri (Platonia insignis Mart), cupuaçu (Theobroma grandiflorum Willd ex-Spreng Schum) e murici (Byrsonima crassifolia L.) processado (aquecido e alcalinizado)
}

Alexandre PORTE 1 , Claudia Moraes REZENDE², Octavio Augusto Ceva ANTUNES ${ }^{3}$, Luciana Helena MAIA ${ }^{4}$

\section{RESUMO}

A literatura científica é pobre a respeito de frutas da Amazônia, como o murici, e suas características químicas devem ser estudadas. Por isso, esta pesquisa teve por proposta determinar o perfil aminoacídico das polpas de bacuri, cupuaçu e murici sob diferentes valores de $\mathrm{pH}\left(3,3,5,8,8,0\right.$ e 12,0), sem aquecimento ou com aquecimento por 12 horas $/ 100{ }^{\circ} \mathrm{C}$ com agitaçáo e refluxo. Valores de $\mathrm{pH}$, glicose, frutose e sacarose também foram determinados nas polpas sem aquecimento. Os nutrientes foram determinados por CLAE (Cromatografia Líquida de Alta Eficiência). As polpas de bacuri, cupuaçu e murici apresentaram valores de $\mathrm{pH} 3,2$, 3,6 e 3,35, respectivamente. A sacarose foi, quantitativamente, o principal carboidrato nas polpas de cupuaçu $(38,34 \%)$ e bacuri $(36,93 \%)$, sendo que os teores de frutose e glicose foram similares, tanto na polpa de cupuaçu $(8,93 \%$ e $9,03 \%)$ como na de bacuri $(12,63 \%$ e $11,65 \%)$, respectivamente. Em contraste, a polpa de murici foi quase isenta de sacarose $(0,57 \%)$, mas não de frutose $(11,51 \%)$ ou glicose $(11,39 \%)$. Nas polpas sem aquecimento, os principais aminoácidos foram: ácido glutâmico (46,6 mg/kg), ácido aspártico $(28,8 \mathrm{mg} / \mathrm{kg})$ e arginina $(25,3 \mathrm{mg} / \mathrm{kg})$ na polpa de bacuri; ácido aspártico $(56,3 \mathrm{mg} / \mathrm{kg})$, ácido glutâmico $(44,0 \mathrm{mg} / \mathrm{kg})$ e alanina $(24,2 \mathrm{mg} / \mathrm{kg})$ na polpa de cupuaçu; prolina $(73,5 \mathrm{mg} / \mathrm{kg})$, ácido glutâmico $(23,7 \mathrm{mg} / \mathrm{kg})$ e ácido aspártico $(23,5 \mathrm{mg} / \mathrm{kg}) \mathrm{na}$ polpa de murici. $\mathrm{O}$ aquecimento reduziu as concentraçóes de todos os aminoácidos nas 3 polpas. O meio fortemente alcalino $(\mathrm{pH}$ 12) produziu a maior degradação de aminoácidos. Lisina foi mais sensível ao aquecimento do que outros aminoácidos em pH 12.

PALAVRAS-CHAVE: aquecimento, aminoácidos, pH, cromatografia líquida de alta eficiência, polpa de fruta

\section{Amino acids reduction in processed (heated and alkalinized) pulps of bacuri (Platonia insignis Mart), cupuaçu (Theobroma grandiflorum Willd ex- Spreng Schum) and murici (Byrsonima crassifolia L.)}

\begin{abstract}
Scientific literature presents few studies about fruits of the Amazonia, like murici, and yours chemical characteristics should be studied. Therefore, amino acid profiles of the bacuri, cupuaçu and murici pulps were determined under different values of $\mathrm{pH}(3.3,5.8,8.0$ and 12.0) with heating ( 12 hours $/ 100^{\circ} \mathrm{C}$, with stirring and refluxing) or without heating. Glucose, fructose, sucrose and $\mathrm{pH}$ values also were obtained in the pulps without heating. All nutrients were analised by HPLC. The pHs were: 3.2,3.6 and 3.35 in the bacuri, cupuaçu and murici pulps, respectively. Sucrose (38.34\% and $36.93 \%)$ was the major carbohydrate while fructose $(8.93 \%$ and $12.63 \%)$ and glucose $(9.03 \%$ and $11.65 \%)$ shown similar percentages in the cupuaçu and bacuri pulps. Murici pulp was almost free of sucrose $(0.57 \%)$, but not of fructose $(11.51 \%)$ or glucose $(11.39 \%)$. In the pulps without heating the major amino acids were: glutamic acid $(46.6 \mathrm{mg} / \mathrm{kg})$, aspartic acid $(28.8 \mathrm{mg} / \mathrm{kg})$ and arginine $(25.3 \mathrm{mg} / \mathrm{kg})$ in the bacuri pulp; aspartic acid $(56.3 \mathrm{mg} / \mathrm{kg})$, glutamic acid $(44.0 \mathrm{mg} / \mathrm{kg})$ and alanine $(24.2 \mathrm{mg} / \mathrm{kg})$ in the cupuaçu pulp; proline $(73.5 \mathrm{mg} / \mathrm{kg})$, glutamic acid $(23.7 \mathrm{mg} / \mathrm{kg})$ and aspartic acid $(23.5 \mathrm{mg} / \mathrm{kg})$ in the murici pulp. The heating of the 3 pulps decresead the concentration of all amino acids. The medium strongly alkaline $(\mathrm{pH} 12)$ produced more degradation of the amino acids than others $\mathrm{pHs}$. Lysine was more sensible to the heating than others amino acids in $\mathrm{pH} 12$.
\end{abstract}

KEYWORDS: heating, amino acids, $\mathrm{pH}$, high performance liquid chromatography, fruit pulp

\footnotetext{
${ }^{1}$ Centro Universitário Augusto Motta .E-mail: alexandre_porte@yahoo.com.br

2 Universidade Federal do Rio de Janeiro. E-mail: crezende@iq.ufrj.br

${ }^{3}$ Universidade Federal do Rio de Janeiro.E-mail: octavio@iq.ufrj.br

${ }^{4}$ Universidade Federal Rural do Rio de Janeiro.E-mail: maia2004@yahoo.com.br
} 


\section{INTRODUÇÃO}

O bacuri (Platonia insignis Mart) da família Gutiferacea, tem o tamanho aproximado de uma laranja, a polpa é branca, acre e doce, com aroma agradável. É nativo da região amazônica, sendo consumido in natura, como suco, sorvete, doce, geléia, néctares, recheio de chocolate e iogurte com aroma bacuri (Borges e Rezende 2000; Franco 2004; Bezerra et al. 2005; Muniz et al. 2006).

O cupuaçu (Theobroma grandiflorum Willd ex-Spreng Schum) da família Sterculiaceae, apresenta forma oval, casca marrom, dura e polpa branca amarelada. Seu sabor é agradável, intenso, agridoce e considerado exótico. É consumido fresco, como suco, sorvetes, licores, compotas, geléias, tortas, néctar enlatado, bombons e biscoitos (Fonseca et al.1990; Franco 2004).

O murici (Byrsonima crassifolia L.) da família Malpighiaceae, é uma pequena fruta tropical de intenso aroma frutal e semelhante a queijo rançoso (Rezende e Fraga 2003).

As frutas nativas da regiâo amazônica e seus derivados vêm se tornando cada vez mais populares no Brasil e têm despertado o interesse internacional. Não obstante, a literatura científica acerca de algumas delas é escassa e demanda mais estudos a respeito de suas características químicas. Por isso, o objetivo deste trabalho foi determinar nas polpas de três frutas nativas da região amazônica (bacuri, cupuaçu e murici): o pH; os teores de sacarose, de frutose e de glicose; e a composiçáo de L-aminoácidos antes e após aquecimento sob diferentes valores de $\mathrm{pH}$.

\section{MATERIAL E MÉTODOS}

As polpas congeladas foram obtidas do comércio na cidade de Belém - PA e transportadas via área acondicionadas em isolantes térmicos até o laboratório, onde foram armazenadas $\mathrm{a}-18{ }^{\circ} \mathrm{C}$ em freezer (Electrolux, Brasil). Para a realização das análises, elas foram descongeladas a temperatura ambiente e após atingirem $25^{\circ} \mathrm{C}$ foram pesadas fraçóes de $100 \mathrm{~g} \mathrm{em}$ balança de precisão (Coleman, Brasil).

Cada fração foi transferida para balâo de fundo redondo de 250 $\mathrm{mL}$ ou recipiente próprio do liofilizador Thermo Savant (Thermo Savant, Estados Unidos da América), modelo Micromodulo 115 e recongelada em gelo seco ou nitrogênio líquido, sob agitação contínua e circular, de forma a distribuir o mais uniformemente possível o conteúdo interno nas paredes e fundo da vidraria.

O processo de liofilizaçáo durou cerca de 12 horas.

Todas as análises seguintes foram realizadas com polpas reconstituídas. Para isso $10 \mathrm{~g}$ de cada polpa de bacuri, murici e cupuaçu liofilizadas foram reconstituídas com 90 $\mathrm{mL}$ de tampão fosfato aquoso [fosfato de sódio monobásico $\left(\mathrm{NaH}_{2} \mathrm{PO}_{4} \cdot \mathrm{H}_{2} \mathrm{O}\right)$ e fosfato de sódio dibásico heptahidratado $\left.\left(\mathrm{Na}_{2} \mathrm{HPO}_{4} .7 \mathrm{H}_{2} \mathrm{O}\right)\right]$. A proporção entre os sais no tampão variou conforme o $\mathrm{pH}$ desejado da solução, $\mathrm{pH}$ 3,3, 5,8, 8,0 e 12,0. Eventuais correçóes do $\mathrm{pH}$ foram realizadas com ácido clorídrico $(\mathrm{HCl})$ 0,1 M ou hidróxido de sódio $(\mathrm{NaOH}) 5 \%$.

A medição do $\mathrm{pH}$ foi realizada em duplicata e a média aritmética registrada como valor final usando medidor de $\mathrm{pH}$ (Digimed, Brasil) após calibração com padrôes de $\mathrm{pH} 4,0 \mathrm{e}$ 7,0, conforme recomendado pelo manual do fabricante.

Os sistemas contendo as $100 \mathrm{~g}$ de cada polpa foram aquecidos a pressão ambiente, usando a temperatura de 100 ${ }^{\circ} \mathrm{C}$, durante 12 horas, sob agitação em agitador magnético com aquecimento (Tecnal, Brasil) e refluxo constante. Após as 12 horas de reação, os conteúdos dos balôes foram resfriados em água corrente até temperatura ambiente e novamente liofilizados. As análises foram realizadas em duplicatas.

Para a análise de aminoácidos, as amostras reliofilizadas foram desengorduradas com hexano e hidrolizadas em ampolas de vidro com $1 \mathrm{mg}$ de proteína/mL de $\mathrm{HCl} 6 \mathrm{~N}$, seladas sob $\mathrm{N}_{2}$ e vácuo e deixados em estufa de secagem (Fanem, Brasil) por 22 horas a $105^{\circ} \mathrm{C}$. Alíquotas do hidrolisado foram tomadas e levadas para a evaporação do ácido, em dessecador sob vácuo constante por 12 horas, com sílica recém ativada. As amostras foram ressuspendidas em $\mathrm{HCl} 20 \mathrm{mM}$, tampão Borato $(\mathrm{pH}$ 8,8) e logo depois foi adicionada uma solução de AMQ (carbamato de 6-aminoquinolil-N-hidroxisuccinimidila), sendo que a reação foi completa com aquecimento à $55^{\circ} \mathrm{C}$ por 10 minutos. As amostras já derivatizadas foram, então, transferidas para frascos de injetor automático e analisadas por CLAE (Cromatografia Líquida de Alta Eficiência). O cromatógrafo utilizado foi Waters Alliance 2695 (Waters, Estados Unidos da América), com detetores de fluorescência 2475 e de arranjo de fotodiodos 2996 (PDA) em linha. Utilizou-se uma coluna Nova-Pak C18, 3,9 × 150 mm, de 4 $\mathrm{mm}$ (Waters, Estados Unidos da América), a $37^{\circ} \mathrm{C}$. Foi feito um gradiente ternário, composto por tampão acetato $(\mathrm{pH}$ 5,05), acetonitrila e água. Os cromatogramas foram extraídos no PDA a $254 \mathrm{~nm}$, enquanto o detector de fluorescência foi ajustado em $250 \mathrm{~nm}$ e $395 \mathrm{~nm}$ como comprimento de excitação e emissão, respectivamente, sendo 40 minutos o tempo de corrida.

Para a análise dos açúcares sacarose, glicose e frutose, foram pesados $1 \mathrm{~g}$ de cada polpa liofilizada em balóes de $25 \mathrm{~mL}$ e, a seguir, estas amostras foram solubilizadas em água deionizada Milli-Q (Millipore Corporation, Estados Unidos da América) levadas ao banho de ultrasom (Cole-Parmer, Estados Unidos da América) por 20 minutos e completado o volume do baláo. A solução foi filtrada e um volume de $20 \mathrm{~mL}$ foi injetado, com tempo de corrida de 20 minutos, ordem de eluiçấo: sacarose, glicose e frutose, detetor de índice de refração, temperatura do detetor de $45^{\circ} \mathrm{C}$, fase móvel: água ultrapura e temperatura do injetor de $10^{\circ} \mathrm{C}$. 
A avaliação dos teores de aminoácidos das polpas de frutas foi feita por Delineamento Inteiramente Casualizado (DIC) com cinco tratamentos (controle, $\mathrm{pHs} 3,3 ; 5,8$; 8,0 e 12,0) e duas repetiçôes. Os dados obtidos foram submetidos à análise de variância e quando significativo, foi aplicado o teste de Tukey ao nível de 5\% de probabilidade para comparaçáo entre as médias, utilizando o programa estatístico ASSISTAT versão 7.3 beta (Silva e Azevedo 2002).

\section{RESULTADOS E DISCUSSÃO}

As polpas de bacuri, cupuaçu e murici apresentaram valores de $\mathrm{pH}$ iguais a 3,2, 3,6 e 3,35, respectivamente. Carvalho et al. (2002) determinou na polpa de bacuri um valor maior para o $\mathrm{pH}$, igual a 3,48.

Os três principais aminoácidos encontrados nas polpas in natura (sem aquecimento) de bacuri (Tabela 1) foram: ácido glutâmico (46,6 mg de aminoácido/kg de polpa, ácido aspártico (28,8 $\mathrm{mg}$ de aminoácido/kg de polpa) e arginina (25,3 $\mathrm{mg}$ de aminoácido/kg de polpa). No cupuaçu (Tabela 2) foram: ácido aspártico $(56,3 \mathrm{mg}$ de aminoácido $/ \mathrm{kg}$ de polpa), ácido glutâmico (44,0 $\mathrm{mg}$ de aminoácido/kg de polpa) e alanina ( $24,2 \mathrm{mg}$ de aminoácido/kg de polpa). E no murici (Tabela 3) foram: prolina (73,5 $\mathrm{mg}$ de aminoácido/ $\mathrm{kg}$ de polpa), ácido glutâmico (23,7 mg de aminoácido/kg de polpa) e ácido aspártico $(23,5 \mathrm{mg}$ de aminoácido/kg de polpa).

Tabela 1 - Teores de aminoácidos $(\mathrm{mg} / \mathrm{kg})$ da polpa de bacuri in natura (controle) e da polpa aquecida em diferentes pHs.

\begin{tabular}{lccccc}
\hline \multirow{2}{*}{ Aminoácidos } & \multicolumn{5}{c}{ Tratamentos } \\
\cline { 2 - 6 } & Controle & $\mathrm{pH} 3,3$ & $\mathrm{pH} 5,8$ & $\mathrm{pH} 8,0$ & $\mathrm{pH} \mathrm{12,0}$ \\
\hline ASP & $28,8 \mathrm{a}$ & $2,5 \mathrm{~b}$ & $2,5 \mathrm{~b}$ & $2,3 \mathrm{~b}$ & $2,1 \mathrm{~b}$ \\
SER & $16,0 \mathrm{a}$ & $1,3 \mathrm{~b}$ & $0,3 \mathrm{~b}$ & $1,2 \mathrm{~b}$ & $0,6 \mathrm{~b}$ \\
GLU & $46,6 \mathrm{a}$ & $3,9 \mathrm{~b}$ & $3,8 \mathrm{~b}$ & $3,5 \mathrm{~b}$ & $3,3 \mathrm{~b}$ \\
GLY & $13,5 \mathrm{a}$ & $0,9 \mathrm{~b}$ & $0,9 \mathrm{~b}$ & $0,9 \mathrm{~b}$ & $0,8 \mathrm{~b}$ \\
HIS & $7,20 \mathrm{a}$ & $0,5 \mathrm{~b}$ & $0,6 \mathrm{~b}$ & $0,5 \mathrm{~b}$ & $0,4 \mathrm{~b}$ \\
ARG & $25,3 \mathrm{a}$ & $1,4 \mathrm{~b}$ & $1,3 \mathrm{bc}$ & $0,5 \mathrm{bc}$ & $0,4 \mathrm{c}$ \\
THR & $12,8 \mathrm{a}$ & $0,9 \mathrm{~b}$ & $1,0 \mathrm{~b}$ & $0,9 \mathrm{~b}$ & $0,3 \mathrm{c}$ \\
ALA & $16,7 \mathrm{a}$ & $1,4 \mathrm{~b}$ & $1,4 \mathrm{~b}$ & $1,2 \mathrm{~b}$ & $1,2 \mathrm{~b}$ \\
PRO & $11,4 \mathrm{a}$ & $0,4 \mathrm{~b}$ & $0,5 \mathrm{~b}$ & $0,5 \mathrm{~b}$ & $0,4 \mathrm{~b}$ \\
TYR & $10,9 \mathrm{a}$ & $1,0 \mathrm{~b}$ & $1,2 \mathrm{~b}$ & $1,0 \mathrm{~b}$ & $0,9 \mathrm{~b}$ \\
VAL & $17,3 \mathrm{a}$ & $1,5 \mathrm{~b}$ & $1,5 \mathrm{~b}$ & $1,3 \mathrm{~b}$ & $1,2 \mathrm{~b}$ \\
LYS & $20,3 \mathrm{a}$ & $1,3 \mathrm{~b}$ & $1,3 \mathrm{~b}$ & $0,6 \mathrm{bc}$ & $0,5 \mathrm{c}$ \\
ILE & $13,4 \mathrm{a}$ & $1,3 \mathrm{~b}$ & $1,4 \mathrm{~b}$ & $1,2 \mathrm{~b}$ & $1,0 \mathrm{~b}$ \\
LEU & $22,8 \mathrm{a}$ & $2,0 \mathrm{~b}$ & $2,0 \mathrm{~b}$ & $1,8 \mathrm{~b}$ & $1,6 \mathrm{~b}$ \\
PHE & $12,4 \mathrm{a}$ & $1,3 \mathrm{~b}$ & $1,4 \mathrm{~b}$ & $1,2 \mathrm{~b}$ & $1,0 \mathrm{~b}$ \\
\hline
\end{tabular}

a,b,c: Médias na mesma linha com letras diferentes, diferem entre si significativamente pelo teste de Tukey a $5 \%$ de probabilidade. Médias de duplicatas. ASP - ácido

aspártico; SER - serina; GLU - ácido glutâmico; GLY - glicina; HIS - histidina; ARG arginina; THR - treonina; ALA - alanina; PRO - prolina; TYR - tirosina; VAL - valina; LYS - lisina; ILE - isoleucina; LEU - leucina; PHE - fenilalanina
Tabela 2 - Teores de aminoácidos (mg/kg) da polpa de cupuaçu in natura (controle) e da polpa aquecida em diferentes pHs.

\begin{tabular}{|c|c|c|c|c|c|}
\hline \multirow{2}{*}{ Aminoácidos } & \multicolumn{5}{|c|}{ Tratamentos } \\
\hline & Controle & $\mathrm{pH} 3,3$ & $\mathrm{pH} 5,8$ & $\mathrm{pH} 8,0$ & $\mathrm{pH} 12,0$ \\
\hline ASP & $44,0 \mathrm{a}$ & $5,9 b$ & $6,6 b$ & $4,3 b$ & $1,8 b$ \\
\hline SER & $16,2 a$ & $1,5 b c$ & $2,2 b$ & $1,5 b c$ & $0,6 c$ \\
\hline GLU & $56,3 a$ & $6,4 b$ & $7,2 b$ & $5,4 b$ & $0,6 c$ \\
\hline GLY & $12,4 a$ & $1,0 b c$ & $1,4 b$ & $1,0 \mathrm{bc}$ & $0,5 c$ \\
\hline HIS & $7,7 a$ & $0,6 b$ & $0,7 \mathrm{~b}$ & $0,4 b c$ & $0,2 c$ \\
\hline ARG & $21,2 \mathrm{a}$ & $1,5 b c$ & $2,0 \mathrm{~b}$ & $0,7 \mathrm{~cd}$ & $0,4 d$ \\
\hline THR & $15,0 \mathrm{a}$ & $1,1 b$ & $1,6 b$ & $1,1 b$ & $0,3 b$ \\
\hline ALA & $24,2 \mathrm{a}$ & $2,5 b c$ & $2,9 b$ & $2,0 \mathrm{bc}$ & $0,9 c$ \\
\hline PRO & $15,8 \mathrm{a}$ & $0,6 b$ & $1,0 \mathrm{~b}$ & $0,8 b$ & $0,4 b$ \\
\hline TYR & $12,8 \mathrm{a}$ & $1,1 b c$ & $1,7 \mathrm{~b}$ & $1,1 b c$ & $0,5 c$ \\
\hline VAL & $19,2 \mathrm{a}$ & $1,8 b c$ & $2,5 b$ & $1,7 \mathrm{bc}$ & $0,8 \mathrm{c}$ \\
\hline LYS & $17,1 \mathrm{a}$ & $1,5 b c$ & $1,9 b$ & $0,9 \mathrm{~cd}$ & $0,4 d$ \\
\hline ILE & $13,6 a$ & $1,3 b$ & $1,9 b$ & $1,2 b$ & $0,6 b$ \\
\hline LEU & $23,9 a$ & $2,2 b c$ & $3,8 b$ & $2,2 b c$ & $1,0 \mathrm{c}$ \\
\hline PHE & $15,4 \mathrm{a}$ & $1,5 b c$ & $2,2 b$ & $1,5 b c$ & $0,7 \mathrm{c}$ \\
\hline
\end{tabular}

a,b,c,d : Médias na mesma linha com letras diferentes, diferem entre si

significativamente pelo teste de Tukey a $5 \%$ de probabilidade. Médias de duplicatas. ASP - ácido aspártico; SER - serina; GLU - ácido glutâmico; GLY - glicina; HIS - histidina; ARG - arginina; THR - treonina; ALA - alanina; PRO - prolina; TYR tirosina; VAL - valina; LYS - lisina; ILE - isoleucina; LEU - leucina; PHE - fenilalanina

Tabela 3 - Teores de aminoácidos $(\mathrm{mg} / \mathrm{kg})$ da polpa de murici in natura (controle) e da polpa aquecida em diferentes pHs.

\begin{tabular}{lccccc}
\hline & \multicolumn{5}{c}{ Tratamentos } \\
\cline { 2 - 6 } Aminoácidos & Controle & pH 3,3 & pH 5,8 & pH 8,0 & pH 12,0 \\
\cline { 2 - 6 } & $23,5 \mathrm{a}$ & $2,5 \mathrm{~b}$ & $2,2 \mathrm{~b}$ & $1,9 \mathrm{~b}$ & $1,3 \mathrm{~b}$ \\
\hline ASP & $14,2 \mathrm{a}$ & $1,5 \mathrm{~b}$ & $1,3 \mathrm{bc}$ & $1,1 \mathrm{bc}$ & $0,5 \mathrm{c}$ \\
SER & $23,7 \mathrm{a}$ & $2,7 \mathrm{~b}$ & $2,3 \mathrm{~b}$ & $2,0 \mathrm{~b}$ & $1,6 \mathrm{~b}$ \\
GLU & $10,3 \mathrm{a}$ & $1,0 \mathrm{~b}$ & $0,9 \mathrm{~b}$ & $0,8 \mathrm{~b}$ & $0,6 \mathrm{~b}$ \\
GLY & $8,5 \mathrm{a}$ & $0,9 \mathrm{~b}$ & $0,7 \mathrm{bc}$ & $0,7 \mathrm{bc}$ & $0,4 \mathrm{c}$ \\
HIS & $13,5 \mathrm{a}$ & $1,1 \mathrm{~b}$ & $1,1 \mathrm{~b}$ & $0,9 \mathrm{bc}$ & $0,3 \mathrm{c}$ \\
ARG & $13,6 \mathrm{a}$ & $1,1 \mathrm{~b}$ & $0,9 \mathrm{bc}$ & $0,8 \mathrm{bc}$ & $0,3 \mathrm{c}$ \\
THR & $15,0 \mathrm{a}$ & $1,6 \mathrm{~b}$ & $1,4 \mathrm{~b}$ & $1,3 \mathrm{~b}$ & $0,7 \mathrm{~b}$ \\
ALA & $73,5 \mathrm{a}$ & $5,1 \mathrm{~b}$ & $4,7 \mathrm{~b}$ & $4,6 \mathrm{~b}$ & $0,9 \mathrm{c}$ \\
PRO & $8,9 \mathrm{a}$ & $1,2 \mathrm{~b}$ & $1,0 \mathrm{~b}$ & $1,0 \mathrm{~b}$ & $0,8 \mathrm{~b}$ \\
TYR & $15,5 \mathrm{a}$ & $1,7 \mathrm{~b}$ & $1,5 \mathrm{~b}$ & $1,3 \mathrm{~b}$ & $0,9 \mathrm{~b}$ \\
VAL & $18,5 \mathrm{a}$ & $2,0 \mathrm{~b}$ & $1,6 \mathrm{~b}$ & $1,2 \mathrm{bc}$ & $0,5 \mathrm{c}$ \\
LYS & $11,3 \mathrm{a}$ & $1,4 \mathrm{~b}$ & $1,2 \mathrm{~b}$ & $1,1 \mathrm{~b}$ & $0,8 \mathrm{~b}$ \\
ILE & $19,3 \mathrm{a}$ & $2,0 \mathrm{~b}$ & $1,8 \mathrm{~b}$ & $1,6 \mathrm{~b}$ & $1,2 \mathrm{~b}$ \\
LEU & $10,9 \mathrm{a}$ & $1,4 \mathrm{~b}$ & $1,3 \mathrm{~b}$ & $1,2 \mathrm{~b}$ & $0,9 \mathrm{~b}$ \\
\hline PHE & &
\end{tabular}

a,b,c : Médias na mesma linha com letras diferentes, diferem entre si significativamente pelo teste de Tukey a $5 \%$ de probabilidade. Médias de duplicatas. ASP - ácido aspártico; SER - serina; GLU - ácido glutâmico; GLY - glicina; HIS - histidina; ARG - arginina; THR - treonina; ALA - alanina; PRO - prolina; TYR tirosina; VAL - valina; LYS - lisina; ILE - isoleucina; LEU - leucina; PHE - fenilalanina 
O triptofano, por ser destruído no meio ácido durante o preparo da amostra para a análise, não foi determinado.

A presença de ácido aspártico e ácido glutâmico entre os aminoácidos majoritários ocorre em diferentes classes de alimentos, como na erva cominho (Cuminum cyminum L.), em uma espécie de cogumelo comestível (Hypsizygus marmoreus (Peck) H. E. Bigelow), em presuntos (Badr e Georgiev 1990; Harada et al. 2003; Quaresma et al. 2003) e em palmito de pupunha (Bactris gasipaes Kunth) (Yuyama et al. 2003).

Os resultados determinados nesta pesquisa diferem daqueles relatados por Rogez et al. (2004), nos quais os principais aminoácidos sáo a lisina no bacuri e a alanina no cupuaçu, embora a alanina tenha sido bastante abundante ( $3^{\circ}$ maior) na polpa de cupuaçu da pesquisa atual.

Infelizmente, a metodologia usada por Rogez et al. (2004) não permite a dosagem de ácido aspártico isolado da asparagina assim como do ácido glutâmico separado da glutamina, dificultando a comparaçáo dos resultados entre ambos os trabalhos.

$\mathrm{O}$ aquecimento das polpas reduziu significativamente os teores dos aminoácidos.

$\mathrm{Na}$ polpa aquecida de bacuri, embora tenha havido diminuiçáo nos teores dos aminoácidos, não houve diferença entre os tratamentos realizados em diferentes valores de $\mathrm{pH}$, exceto para três aminoácidos: arginina, treonina e lisina. Para estes aminoácidos, o aquecimento em $\mathrm{pH} 12,0$ promoveu maior degradaçáo do que em pH 3,3 (Tabela 1).

Treonina, prolina, isoleucina e ácido aspártico foram os únicos aminoácidos que tiveram seus teores igualmente reduzidos em todos os tratamentos com diferentes valores de $\mathrm{pH}$, na polpa de cupuaçu aquecida. $\mathrm{Na}$ maioria dos aminoácidos, houve diferença entre o pH 5,8 e o 12,0, sendo que o $\mathrm{pH}$ alcalino, de novo promoveu maiores perdas nos teores dos aminoácidos. Para arginina e lisina também foi significativa a perda em seus teores em $\mathrm{pH} 12,0$, se comparada aos tratamentos em pH 3,3 e 5,8 (Tabela 2).

A degradaçáo foi estatisticamente igual para a maioria dos aminoácidos na polpa de murici aquecida em todos os tratamentos de $\mathrm{pH}$. Para serina, histidina e treonina o $\mathrm{pH} 12,0$ afetou mais do que o $\mathrm{pH} 3,3$. Para arginina e lisina o $\mathrm{pH}$ 12,0 afetou mais do que o $\mathrm{pH} 3,3$ e o $\mathrm{pH} 5,8$, e, para a prolina, o aminoácido mais abundante, o $\mathrm{pH} 12,0$ reduziu o teor mais do que os outros três valores de $\mathrm{pH}$ (Tabela 3 ).

No pH 12,0 ocorreram as maiores perdas de aminoácidos em todas as polpas aquecidas (Tabelas 1, 2 e 3).

A lisina, apesar de ser um aminoácido básico, foi o aminoácido mais sensível em $\mathrm{pH}$ fortemente alcalino $(12,0)$, apresentando perdas significativas em todas as polpas (Tabelas 1, 2 e 3). A treonina também apresentou este mesmo comportamento frente ao meio básico, exceto na polpa de cupuaçu (Tabela 2), cujo pH não afetou a diminuição do seu teor.

Pela Tabela 4, verifica-se que a sacarose foi o principal açúcar no bacuri e no cupuaçu, enquanto no murici seu teor foi muito baixo. Os teores de glicose e frutose foram próximos nas três polpas.

Os resultados encontrados para a polpa de bacuri e cupuaçu (Tabela 4) confirmam aqueles relatados por Rogez et al. (2004), nos quais sacarose é o principal açúcar, sendo que a glicose e a frutose estão presentes em teores bastante próximos.

Tabela 4 -Teores de açúcares das polpas de bacuri, cupuaçu e murici (\% b.s.)

\begin{tabular}{lccc}
\hline Açúcares & Bacuri & Cupuaçu & Murici \\
\hline Glicose & $11,65 \pm 1,37$ & $9,03 \pm 0,81$ & $11,39 \pm 1,25$ \\
Sacarose & $36,93 \pm 2,13$ & $38,84 \pm 2,09$ & $0,57 \pm 0,42$ \\
Frutose & $12,63 \pm 1,02$ & $8,93 \pm 0,86$ & $11,51 \pm 1,56$
\end{tabular}

b.s. - base seca

\section{AGRADECIMENTOS}

Ao CNPq pelo apoio financeiro.

\section{BIBLIOGRAFIA CITADA}

Badr, F.H.; Georgiev, E.V. 1990. Amino acid composition of cumin seed (Cuminum cyminum L.). Food Chemistr., 38: 273-278.

Bezerra, G.S.A.; Maia, G.A.; Figueiredo, R.W.; Souza Filho, M.S.M. 2005. The agro economical potential of bacuri: a review. Boletim CEPPA, 23: 47-58 (in Portuguese, with abstract in English).

Borges, E.S.; Rezende, C.M. 2000. Main aroma constituents of Genipap (Genipa americana L.) and Bacuri (Platonia insignis M.). Journal of Essential Oil Research, 12: 71-74.

Carvalho, J.E.U.; Alves, S.M.; Nascimento, W.M.O.; Muller, C.H. 2002. Physical and chemical characteristics of a seedless bakuri (Platonia insignis mart.) type. Revista Brasileira de Fruticultura, 24: 573-575 (in Portuguese, with abstract in English).

Franco, M.R.B. 2004. Aroma and Flavour of food: current issues. Varela, São Paulo - SP, p. 89-90; 93-99 (in Portuguese).

Fonseca, C.E.L.; Escobar, J.R.; Bueno, D.M. 1990. Variablility of some physical and chemical traits of cupuaçuzeiro fruit. Pesquisa Agropecuária Brasileira, 25: 1079-1084 (in Portuguese, with abstract in English).

Harada, A.; Yoneyama, S.; Doi, S.; Aoyama, M. 2003. Changes in contents of free amino acids and soluble carbohydrates during fruit-body development of Hypsizygus marmoreus. Food Chemistry, 83: 343-347.

Muniz, M.B.; Queiroz, A.J.M.; Figueirêdo, R.M.F. Duarte, M.E.M. 2006. Thermophysical characterization of bacuri pulp. Ciência e Tecnologia de Alimentos, 26: 360-368 (in Portuguese, with abstract in English). 
Quaresma, M.A.G.; Alfaia, C.M.P.M.; Xavier, A.F.A.; Partidário, A.M.C.; Mimoso, M.J.C.; Prates, J.A.M. 2003. Free amino acids profile in short ripening Portuguese dry-cured hams. Revista Portuguesa de Ciências Veterinárias, 98: 19-24 (in Portuguese, with abstract in English).

Rezende, C.M.; Fraga, S.R.G. 2003. Chemical and aroma determination of the pulp and seeds of murici (Byrsonima crassifolia L.). Journal of the Brazilian Chemical Society, 14: 425-428.

Rogez, H.; Buxant, R.; Mignolet, E.; Souza, J.N.S.; Silva, E.M.; Larondelle, Y. 2004. Chemical composition of the pulp of three tipical Amazonian fruits: araça-boi (Eugenia stipitata), bacuri (Platonia insignis) and cupuaçu (Theobroma grandiflorum). European Journal of Research and Technology, 218: 380-384.
Silva, F.A.S.E.; Azevedo, C.A.V. 2002. Assistat computational program version for the windows operating system. Revista Brasileira de Produtos Agroindustriais, 4: 71-78.

Yuyama, L.K.O.; Aguiar, J.P.L.; Yuyama, K.; Clement, C.R.; Macedo, S.H.M.; Fávaro, D.I.T.; Afonso, C.; Vasconcelos, M.B.A.; Pimentel, S.A.; Badolato, E.S.G.; Vannucchi, H. 2003. Chemical composition of the fruit mesocarp of three peach palm (Bactris gasipaes) populatíons grown in central Amazonia, Brazil. International Journal of Food Science and Nutrition, 54: 49-56.

Recebido em 27/11/2007

Aceito em 04/01/2010 